\title{
Optimal Homotopy Analysis of MHD Natural Convection Flow of Thixotropic Fluid under Subjection of Thermal Stratification: Boundary Layer Analysis
}

\author{
T. Oreyeni' ${ }^{1}$ E. Omokhuale ${ }^{2}$ \\ ${ }^{1}$ Department of Physical Sciences, Precious Cornerstone University, Ibadan, Nigeria \\ ${ }^{2}$ Department of Mathematical Sciences, Federal University Gusau, Nigeria \\ Email: oreyenit@yahoo.com,emmanuelomokhuale@yahoo.com
}

How to cite this paper: Oreyeni, T. and Omokhuale, E. (2019) Optimal Homotopy Analysis of MHD Natural Convection Flow of Thixotropic Fluid under Subjection of Thermal Stratification: Boundary Layer Analysis. American Journal of Computational Mathematics, 9, 116-131.

https://doi.org/10.4236/ajcm.2019.92009

Received: April 26, 2019

Accepted: June 23, 2019

Published: June 26, 2019

Copyright $\odot 2019$ by author(s) and Scientific Research Publishing Inc. This work is licensed under the Creative Commons Attribution International License (CC BY 4.0).

http://creativecommons.org/licenses/by/4.0/

\begin{abstract}
In this article, the model of a non-Newtonian fluid (Thixotropic) flow past a vertical surface in the presence of exponential space and temperature dependent heat source in a thermally stratified medium is studied. It is assumed that free convection is induced by buoyancy and exponentially decaying internal heat source across the space. The dynamic viscosity is taken to be constant and thermal conductivity of this particular fluid model is assumed to vary linearly with temperature. Thermal stratification has been properly incorporated into the governing equation so that its effect can be revealed and properly reported. The governing partial differential equations describing the model are transformed and parameterized to a system of non-linear ordinary differential equation using similarity transformations. Approximate analytic solutions were obtained by adopting Optimal Homotopy Analysis Method (OHAM). The results show that for both cases of non-Newtonian parameters (Thixotropic) $\left(K_{1}=K_{2}=0\right.$ \& $\left.K_{1}=K_{2}=1.0\right)$, increasing stratification parameters, relate to decreasing in the heat energy entering into the fluid region and thus reducing the temperature of the Thixotropic fluid as it flows.
\end{abstract}

\section{Keywords}

Thixotropic Fluid, Natural Convection, Thermal Stratification, Optimal Homotopy Analysis Method and Boundary Layer Analysis

\section{Introduction}

The study of non-Newtonian fluid flow has attracted the attention of researchers 
over the past years owing to its numerous applications in industry. Some of the applications are encountered in paper production, food processing, glass-fibre and injection moulding, etc. The major difference between a Newtonian and non-Newtonian fluid is that Newtonian fluid, for instance water, does not resist much stress that is applied on them and does not exhibit any sign of strain or deformation unlike non-Newtonian fluid that will quickly exhibit signs of strain when stress is applied. Most of these non-Newtonian fluids are described as "time-dependent" due to the fact that after deformation it takes a fixed time to return to a more viscous state, and likewise the fluid flow is highly dependent on the viscosity. Fluids are referred to as shear thickening when the viscosity increases with the application of the shear stress over time we can categorize rheopetic fluids as shear thickening because they become more viscous over time when shaken or stressed. Fluids are also referred to as shear thinning when the viscosity decreases with the stress over time, thixotropic fluids exhibit this shear thinning property in the sense that, they become less viscous over time when shaken or stressed. In a more simple way, thixotropic fluids are described as fluids that are very thick (viscous) under their normal conditions, but become thin, less viscous and change their flow behavior when pressure is introduced. Many gels and colloids are thixotropic materials. Some natural examples of materials that may be thixotropic are honey, synovial fluid found in joints between some bones, sand, quicksand, clays and drilling muds Hendrickson [1]. In the practical wise, some of implications of the behavior of thixotropic materials are Liquefaction. Liquefaction is referred to as a phenomenon whereby saturated or partially saturated soil substantially loses strength and stiffness in response to an applied stress, usually earthquakes shaking or other sudden changes in stress conditions, causing it to behave like a liquid, that is, it temporarily turns firm ground into a liquid. Although, the impact of liquefaction has being felt some long time ago, but in recent times, a wide destruction made to residential properties in the eastern suburbs and satellite townships of Christchurch, New Zealand during the 2010 Canterbury earthquakes and also the Christchurch earthquakes that occurred in 2011 are caused by Liquefaction Davies et al. [2]. As a result of the complexities encountered working with this fluid, there are virtually very few published works that concentrate on boundary layer flows of the fluids. It was reported in the work of Sadeghy et al. [3] that Harris [4] [5] tried to address the boundary layer flow of thixotropic fluids. It was further reported that, he relied on a simple thixotropic fluid model (the so-called Harris model) to investigate the effects of a fluid's thixotropic behaviour on the characteristics of the momentum boundary layer formed above a fixed plate and also, Hayat et al. [6] investigated MHD flow of Thixotropic fluid with variable thermal conductivity and thermal radiation, it was reported that the non-Newtonian parameters have quite opposite effects on the velocity and temperature distributions.

Convection on the other hand may be classified depending on how the fluid motion is initiated. Natural convection which is also termed buoyant or free convection is a mechanism of heat transportation in which the fluid motion is 
not initiated by an external source such as pump, fan, suction device, but only by fluid density differences in the fluid occurring due to temperature gradients and the driving force for natural convection is buoyancy which is as a result of differences in density. During this process of heating, the density changes in the boundary layer will cause the fluid to rise and be replaced by cooler fluid. Definitely, the cooler fluid will also be heated and rise. Recently, Animasaun [7] [8] discussed the convection which occurs in fluid flow along a vertical surface with a small magnitude of temperature (absolute zero) and an upper horizontal surface of a paraboloid of revolution, modified Boussinesq approximation models for both cases were presented.

Stratification of fluid is a formation of layers and occurs due to temperature variations and concentration differences. This natural process creates a transition zone of a temperature gradient between cold and hot fluid zones and hence in case of vertical natural convection it plays an important role in vertical temperature distribution. The dynamics of thermal stratification is essential for solar engineering because stratification may predict the possibilities of achieving higher energy efficiency. Oreyeni et al. [9] extensively discussed homotopy analysis of MHD free convective micropolar fluid flow along a vertical surface embedded in non-Darcian thermally-stratified medium. It was reported that stratification leads to decline in the velocity and temperature of the fluid respectively and the result is similar to what is observed in the work of Animasaun and Omowaye [10] on upper-convected Maxwell fluid flow with variable thermo-physical properties over a melting surface situated in hot environment subject to thermal stratification and the work of Hayat et al. [11] on thermal and concentration stratifications effects in radiative flow of a Jeffery fluid over a stretching sheet.

Internal energy generation is described as scientific method used in generating heat energy within a body by a chemical, electrical or nuclear process. Eldahab and Aziz [12] investigated the effect of non-uniform heat source with suction-blowing. Muthtamilselvan et al. [13] analyzed effect of non-uniform heat generation on unsteady MHD non-Darcian flow over a vertical stretching surface with variable properties. Crepeau and Clarksean [14] examined a similarity solution for a fluid with an exponentially decaying heat generation term and a constant temperature vertical plate under the assumption that the fluid has an internal volumetric heat generation. An exponentially form is used to account for the internal energy generation term. It was reported that the effect of internal heat generation is important in several applications i.e. reactor safety analysis, fire and combustion studies. In many situations, there may be appreciable temperature difference between the surface and the ambient fluid.

Physical changes of thixotropy which referred to the reversible changes from a flowable fluid to a solid-like elastic gel has been made to occur by changing the temperature. But due to the fact that thixotropic fluid exhibit time-dependent shear thinning property, viscosity of the fluid is assumed to be constant and thermal conductivity is assumed not to be constant and has been taken as function of temperature. A slight increase in the temperature enhances the 
transport phenomena by reducing the viscosity across the momentum boundary layer, hence the heat transfer rate at the wall is also affected. Sharma and Singh [15] presented effects of variable thermal conductivity and heat source/sink on mhd flow near a stagnation point on a linearly stretching sheet. Effects of variable thermal conductivity on heat and mass transfer with Jeffery fluid was investigated by Uwanta and Omokhuale [16]. Prasad et al. [17] discussed the effects of variable fluid properties on the hydromagnetic flow and heat transfer over a non-linearly stretching sheet. Omokhuale et al. [18] studied effect of Jeffery fluid on heat and mass transfer past a vertical porous plate with soret and variable thermal conductivity. Motivated by all the above works and applications, it is of interest to contribute to the body of knowledge by investigating the natural convection flow of thixotropic fluid in a thermally stratified medium and considering cases where the non-Newtonian parameters are properly accounted for. Furthermore, this study examines the characteristics behavior of thixotropic fluid on boundary layer.

\section{Mathematical Formulation}

A steady and incompressible magnetohydrodynamic (MHD) free convective boundary layer flow of thixotropic fluid over a vertical surface in a thermally stratified medium with temperature-dependent thermal conductivity is considered. The motion of an incompressible non-Newtonian fluid is induced by the stretching property of the vertical surface, buoyancy effect which is generated by gradients in the temperature field and space dependent internal heat generation. The flow is assumed to flow in $x$-direction which is along vertical surface and $y$-axis is normal to it. The temperature of the surface $T_{w}$ is held uniform and embedded in a thermally stratified medium of variable ambient temperature $T_{\infty}$ where $\left(T_{w}>T_{\infty}\right)$. A constant magnetic field of strength $B_{o}$ is applied in the $y$-direction. Also the magnetic Reynolds number is assumed to be small so that the induced magnetic field is negligible in comparison to the applied magnetic field.

The equation of continuity can be written as;

$$
\frac{\partial u}{\partial x}+\frac{\partial v}{\partial y}=0
$$

For any incompressible fluid satisfying the thixotropic model with constant viscosity and temperature-dependent thermal conductivity, the momentum and energy equations can be simplified using the usual boundary layer theory approximations and obtain

$$
\begin{aligned}
& u \frac{\partial u}{\partial x}+v \frac{\partial u}{\partial y} \\
= & \frac{\mu}{\rho}\left(\frac{\partial^{2} u}{\partial y^{2}}\right)-\frac{6 R_{1}}{\rho}\left(\frac{\partial u}{\partial y}\right)^{2} \frac{\partial^{2} u}{\partial y^{2}}+\frac{4 R_{2}}{\rho}\left(\frac{\partial u}{\partial y}\right)\left(\frac{\partial^{2} u}{\partial y^{2}}\right)\left(u \frac{\partial^{2} u}{\partial x \partial y}+v \frac{\partial^{2} u}{\partial y^{2}}\right) \\
& +\frac{4 R_{2}}{\rho}\left(\frac{\partial u}{\partial y}\right)^{2}\left(u \frac{\partial^{3} u}{\partial x \partial y^{2}}+v \frac{\partial^{3} u}{\partial y^{3}}+\frac{\partial u}{\partial y^{3}}+\frac{\partial u}{\partial y} \frac{\partial^{2} u}{\partial x \partial y}+\frac{\partial v}{\partial y} \frac{\partial^{2} u}{\partial y^{2}}\right)-\sigma \frac{B_{o}^{2} u}{\rho}+g \beta\left(T-T_{\infty}\right),
\end{aligned}
$$




$$
\begin{aligned}
& u \frac{\partial T}{\partial x}+v \frac{\partial T}{\partial y} \\
& =\frac{1}{\rho C_{p}} \frac{\partial}{\partial y}\left(\kappa(T) \frac{\partial T}{\partial y}\right)+\frac{\kappa a}{\rho C_{p}} \vartheta\left[A\left(T_{w}-T_{0}\right) \exp ^{-y} \sqrt{\frac{a}{\vartheta}}+B\left(T-T_{\infty}\right)\right]
\end{aligned}
$$

Subject to boundary conditions

$$
\begin{gathered}
u=u_{w}(x)=a x, \quad v=v_{w}(x), \quad T=T_{w}, \quad \text { at } y=0, \\
u \rightarrow 0, \quad T \rightarrow T_{\infty}, \quad \text { as } y \rightarrow \infty,
\end{gathered}
$$

In order to justify the variation in the thermo-physical property of the thixotropic fluid as it flows past a vertical heated surface, classical Boussinesq's approximation is adopted such that the temperature at the surface is greater than temperature of the fluid at the free stream. It is valid to consider the mathematical model of temperature dependent thermal conductivity model of Charraudeau [19] as;

$$
\kappa(T)=\kappa^{*}\left[1+\delta\left(T-T_{\infty}\right)\right]
$$

$\kappa^{*}$ are the constant value of the coefficient thermal conductivity at the free stream. Where $u$ and $v$ are components of velocity in $x$ and $y$ directions respectively, $u_{w}(x)$ is the wall shrinking or stretching velocity, $(a>0)$ for stretching, $(a<0)$ for shrinking and $(a=0)$ for static wall, $v_{w}(x)$ is the wall mass flux velocity, $\rho$ is the fluid density, $\vartheta=\frac{\mu}{\rho}$ is the kinematic viscosity, $\sigma$ is the electrical conductivity, $T$ is the fluid temperature in the boundary layer, $T_{\infty}$ is the free stream temperature, $\beta$ is the thermal expansion coefficient, $\alpha=\frac{\kappa}{\rho C_{p}}$ is the thermal diffusivity, $R_{1}$ and $R_{2}$ are the non-Newtonian material constants, $\beta$ is the volumetric coefficient of thermal expansion, $A$ and $B$ are the coefficient of exponentially decaying space and temperature dependent heat source respectively.

The momentum and energy equations can be transformed into the corresponding ordinary differential equations by the following transformations

$$
\eta=\frac{a^{\frac{1}{2}}}{\vartheta^{\frac{1}{2}}} y, \quad \frac{\psi(x, y)}{x a^{\frac{1}{2}} \vartheta^{\frac{1}{2}}}=f(\eta), \quad T-T_{\infty}=\theta(\eta)\left[T_{w}-T_{0}\right]
$$

where $\eta$ is the independent dimensionless similarity variable. Thus $u$ and $v$ are given by $u=a x f^{\prime}(\eta), \quad v=-\sqrt{a \vartheta} f(\eta)$, substituting variables (7) into equations (2)-(5), we obtain the following ordinary differential equations:

$$
\begin{aligned}
& \frac{\mathrm{d}^{3} f}{\mathrm{~d} \eta^{3}}-\left(\frac{\mathrm{d} f}{\mathrm{~d} \eta}\right)^{2}+f(\eta) \frac{\mathrm{d}^{2} f}{\mathrm{~d} \eta^{2}}+K_{1}(x)\left(\frac{\mathrm{d}^{2} f}{\mathrm{~d} \eta^{2}}\right)^{2} \frac{\mathrm{d}^{3} f}{\mathrm{~d} \eta^{3}} \\
& +K_{2}(x) \frac{\mathrm{d} f}{\mathrm{~d} \eta}\left(\frac{\mathrm{d}^{2} f}{\mathrm{~d} \eta^{2}}\right)^{2} \frac{\mathrm{d}^{3} f}{\mathrm{~d} \eta^{3}}-K_{2}(x) f \frac{\mathrm{d}^{2} f}{\mathrm{~d} \eta^{2}}\left(\frac{\mathrm{d}^{3} f}{\mathrm{~d} \eta^{3}}\right)^{2} \\
& -K_{2}(x) f\left(\frac{\mathrm{d}^{2} f}{\mathrm{~d} \eta^{2}}\right)^{2} \frac{\mathrm{d}^{4} f}{\mathrm{~d} \eta^{4}}+K_{2}(x)\left(\frac{\mathrm{d}^{2} f}{\mathrm{~d} \eta^{2}}\right)^{4}-M \frac{\mathrm{d} f}{\mathrm{~d} \eta}+G_{r} \theta
\end{aligned}
$$




$$
[1+\theta \varepsilon] \frac{\mathrm{d}^{2} \theta}{\mathrm{d} \eta^{2}}-P_{r} S_{t} \frac{\mathrm{d} f}{\mathrm{~d} \eta}-P_{r} \theta \frac{\mathrm{d} f}{\mathrm{~d} \eta}+P_{r} f \frac{\mathrm{d} \theta}{\mathrm{d} \eta}+\varepsilon\left(\frac{\mathrm{d} \theta}{\mathrm{d} \eta}\right)^{2}+\left(A \mathrm{e}^{-\eta}+B \theta\right)=0
$$

The corresponding boundary conditions take the form;

$$
\begin{gathered}
f(0)=s, \quad f^{\prime}(0)=1, \quad \theta(0)=1-S_{t}, \quad \text { at } \eta=0, \\
f^{\prime}(\eta) \rightarrow 0, \quad \theta(\eta) \rightarrow 0, \rightarrow 0 \quad \text { as } \eta \rightarrow \infty .
\end{gathered}
$$

In the above equations, primes denote differentiation with respect to $\eta$. The dimensionless velocity and temperature are represented as $f(\eta)$ and $\theta(\eta)$, $P_{r}=\frac{\vartheta}{\alpha}$ is the Prandtl number, $M=\sigma \frac{B_{o}^{2}}{\rho a}$ is the magnetic parameter, $G_{r}=\frac{g \beta\left(T_{w}-T_{\infty}\right)}{a^{2} x}$ is the Modified Thermal Grashof number, $A$ is the space dependent heat source parameter, $S_{t}=\frac{m_{2}}{m_{1}}$ is the thermal stratification parameter, $\varepsilon=\delta\left(T_{w}-T_{0}\right)$ is the temperature-dependent thermal conductivity parameter, $B$ is the temperature dependent heat source parameter, $K_{1}(x)=-\frac{6 R_{1} a^{3} x^{2}}{\rho \vartheta^{2}}$ and $K_{2}(x)=\frac{4 R_{2} a^{4} x^{2}}{\rho \vartheta^{2}}$ are the non-Newtonian parameters.

The dimensionless form of the skin friction coefficient is given as;

$$
\operatorname{Re}_{x}^{1 / 2} C_{f}=f^{\prime \prime}(0)-\frac{K_{1}}{6}\left[f^{\prime \prime}(0)\right]^{3} .
$$

\section{Optimal Homotopy Analysis Solutions}

The analytic solutions of Equations (8) and (9) to the boundary conditions (10) and (11) have been computed by optimal homotopy analytic method (OHAM). Various researchers ([20] [21] [22]) have applied this method to compute flow problems. The suitable initial guesses and the linear operators satisfying the given boundary conditions (10) and (11) for $f(\eta)$ and $\theta(\eta)$ have been carefully selected. The initial guesses for this problem are of the form

$$
f_{o}(\eta)=1+S-\exp (-\eta), \quad \theta_{o}(\eta)=1-S_{t} * \exp (-\eta),
$$

Linear operators $L_{f}$ and $L_{\theta}$ are

$$
\begin{gathered}
L_{f}[f(\eta ; q)]=\frac{\partial^{3} f(\eta ; q)}{\partial \eta^{3}}-\frac{\partial f(\eta ; q)}{\partial \eta} \\
L_{\theta}[\theta(\eta ; q)]=\frac{\partial^{2} \theta(\eta ; q)}{\partial \eta^{2}}-\theta(\eta ; q)
\end{gathered}
$$

The operators $L_{f}$ and $L_{\theta}$ have the following properties

$$
L_{f}\left[C_{1}+C_{2} \exp (-\eta)+C_{3} \exp (\eta)\right]=0, \quad L_{\theta}\left[C_{4} \exp (-\eta)+C_{5}\right]=0
$$

In which $C_{1}, C_{2}, C_{3}, C_{4}$ and $C_{5}$ are constants.

$$
(1-q) L_{f}\left[f(\eta ; q)-f_{o}(\eta)\right]=q \hbar_{f} H_{f}(\eta) N[f(\eta ; q), \theta(\eta ; q)]
$$




$$
(1-q) L_{\theta}\left[\theta(\eta ; q)-\theta_{o}(\eta)\right]=q \hbar_{\theta} H_{\theta}(\eta) N[f(\eta ; q), \theta(\eta ; q)]
$$

Subject to boundary conditions

$$
\begin{gathered}
f(\eta=0 ; q)=s, \quad \frac{\partial f(\eta=0 ; q)}{\partial \eta}=1, \quad \theta(\eta=0 ; q)=1-S_{t} \\
\frac{\partial f(\eta \rightarrow \infty ; q)}{\partial \eta} \rightarrow 0, \quad \theta(\eta \rightarrow \infty) \rightarrow 0
\end{gathered}
$$

where $q$ is the embedding parameters, $\hbar_{f}$ and $\hbar_{\theta}$ the non-zero auxilliary parameters and operators $N_{f}$ and $N_{\theta}$ are given by

$$
\begin{array}{r}
N_{f}[f(\eta, q)]=\frac{\partial^{3} f(\eta ; q)}{\partial \eta^{3}}-\left(\frac{\partial f(\eta ; q)}{\partial(\eta)}\right)^{2}+f(\eta ; q) \frac{\partial^{2} f(\eta ; q)}{\partial \eta^{2}} \\
+K_{1}(x)\left(\frac{\partial^{2} f(\eta, q)}{\partial \eta^{2}}\right)^{2} \frac{\partial^{3} f(\eta, q)}{\partial \eta^{3}}-M \frac{\partial f(\eta, q)}{\partial \eta} \\
K_{2}(x) \frac{\partial f(\eta, q)}{\partial \eta}\left(\frac{\partial^{2} f(\eta, q)}{\partial \eta^{2}}\right)^{2} \frac{\partial^{3} f(\eta, q)}{\partial \eta^{3}}+K_{2}(x)\left(\frac{\partial^{2} f(\eta, q)}{\partial \eta^{2}}\right)^{4} \\
-K_{2}(x) f(\eta, q) \frac{\partial^{2} f(\eta, q)}{\partial \eta^{2}}\left(\frac{\partial^{3} f(\eta, q)}{\partial \eta^{3}}\right)^{2} \\
-K_{2}(x) f(\eta, q)\left(\frac{\partial^{2} f(\eta, q)}{\partial \eta^{2}}\right)^{2} \frac{\partial^{4} f(\eta, q)}{\partial \eta^{4}}+G_{r} \theta(\eta ; q) \\
(1+\theta(\eta ; q) \varepsilon) \frac{\partial^{2} \theta(\eta ; q)}{\partial \eta^{2}}+P_{r} f(\eta ; q) \frac{\partial \theta(\eta ; q)}{\partial \eta}+\varepsilon \frac{\partial \theta(\eta ; q)}{\partial \eta} \frac{\partial \theta(\eta ; q)}{\partial \eta} \\
+\left(A \mathrm{e}^{-\eta}+B \theta(\eta ; q)\right)=0
\end{array}
$$

Obviously, when $q=0$ and $q=1$, zero order of deformation equations (17)-(18) leads to

$$
\begin{aligned}
& f(\eta ; 0)=f_{o}(\eta), \quad f(\eta ; 1)=f_{o}(\eta) \\
& \theta(\eta ; 0)=\theta_{o}(\eta), \quad \theta(\eta ; 1)=\theta_{o}(\eta)
\end{aligned}
$$

Expanding $f(\eta ; q)$ and $\theta(\eta ; q)$ in Taylor series with respect to the embedding parameter $q$,

$$
\begin{aligned}
& f(\eta ; q)=f_{o}(\eta)+\sum_{m=1}^{\infty} f_{m}(\eta) q^{m} \quad \text { where } f_{m}(\eta)=\left.\frac{1}{m !} \frac{\partial^{m} f(\eta ; q)}{\partial q^{m}}\right|_{q=0} \\
& \theta(\eta ; q)=\theta_{o}(\eta)+\sum_{m=1}^{\infty} \theta_{m}(\eta) q^{m} \quad \text { where } \theta_{m}(\eta)=\left.\frac{1}{m !} \frac{\partial^{m} \theta(\eta ; q)}{\partial \eta^{m}}\right|_{q=0}
\end{aligned}
$$

The auxilliary parameters $\hbar_{f}$ and $\hbar_{\theta}$ are properly chosen so that the series Equations (17)-(18) converge at $q=1$. Hence,

$$
\begin{aligned}
& f(\eta ; q)=f_{o}(\eta)+\sum_{m=1}^{\infty} f_{m}(\eta) q^{m} \\
& \theta(\eta ; q)=\theta_{o}(\eta)+\sum_{m=1}^{\infty} \theta_{m}(\eta) q^{m}
\end{aligned}
$$


If we denote the special solutions $f_{m}^{*}$ and $\theta_{m}^{*}$ then the general solutions $f_{m}$ and $\theta_{m}$ are

$$
\begin{gathered}
f_{m}(\eta)=f_{m}^{*}(\eta)+C_{1}+C_{2} \mathrm{e}^{\eta}+C_{3} \mathrm{e}^{-\eta}, \\
\theta_{m}(\eta)=\theta_{m}^{*}(\eta)+C_{4} \mathrm{e}^{\eta}+C_{5} \mathrm{e}^{-\eta} .
\end{gathered}
$$

Following the rule of solution expression, the rule of coefficient ergodicity and the rule of solution existence as discussed in [23] and [24] we choose auxiliary functions as

$$
H_{f}=H_{p}=H_{\theta}=1
$$

In order to analyze the salient characteristics of the problem, the results are presented in Figure 1(a)-Figure 8(b). The effect of non-Newtonian parameters $K_{1}=K_{2}$ on velocity and temperature profiles are revealed in Figure 1(a), Figure 1(b) and Figure 2(a), Figure 2(b) for different cases of stratification

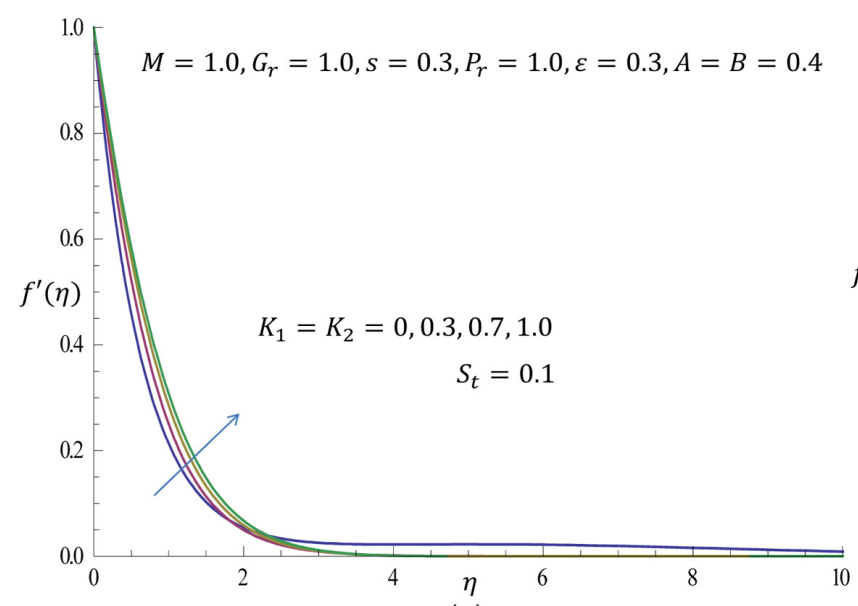

(a)

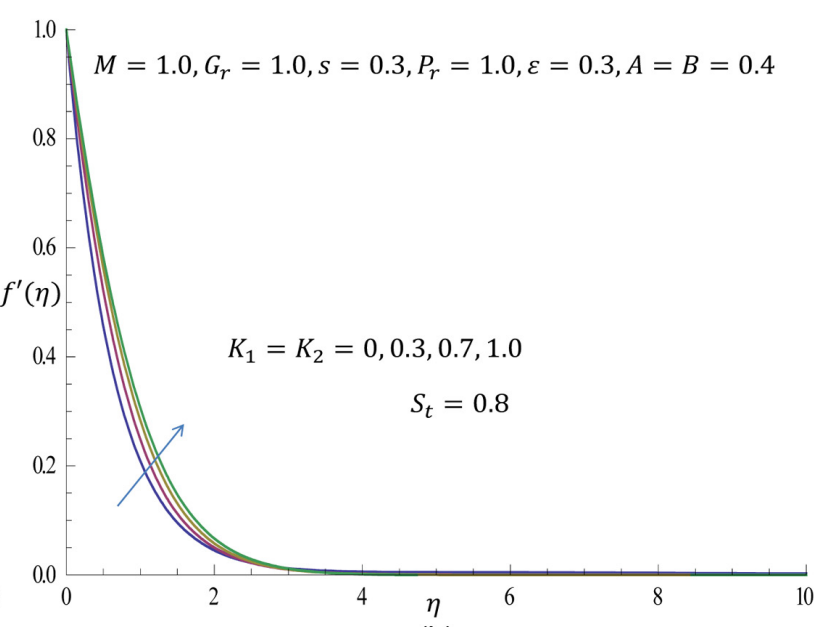

(b)

Figure 1. (a) Effect of non-Newtonian parameters $K_{1}=K_{2}$ on velocity profile when $S_{t}=0.1$ (b) Effect of non-Newtonian parameters $K_{1}=K_{2}$ on velocity profile when $S_{t}=0.8$.

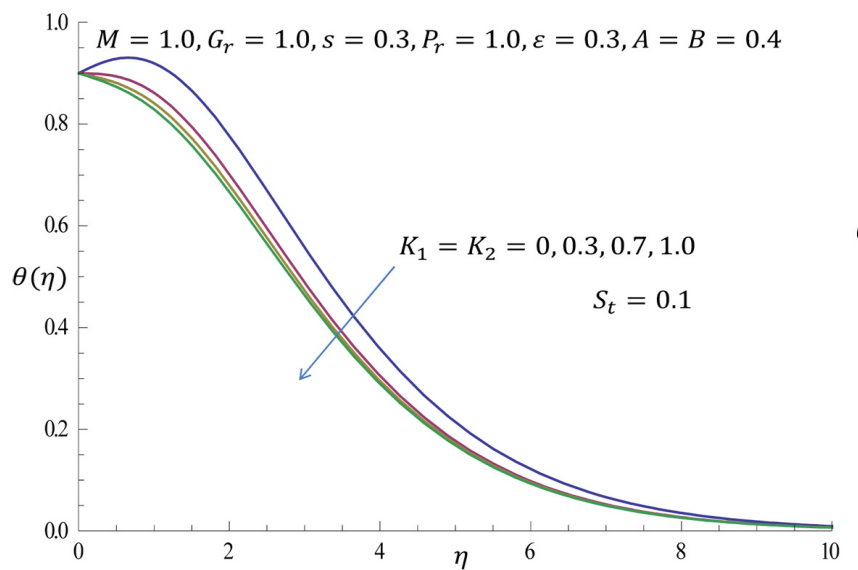

(a)

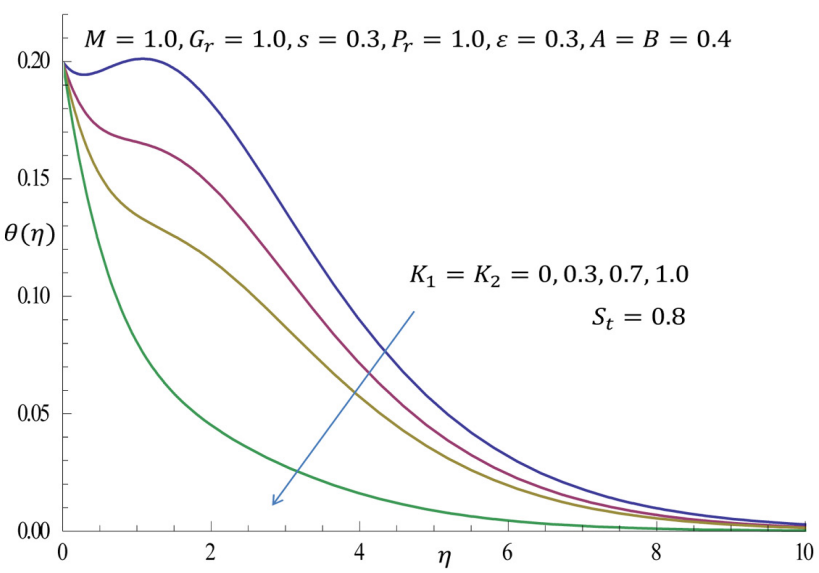

(b)

Figure 2. (a) Effect of non-Newtonian parameters $K_{1}=K_{2}$ on temperature profile when $S_{t}=0.1$ (b) Effect of non-Newtonian parameters $K_{1}=K_{2}$ temperature profile when $S_{t}=0.8$. 
$S_{t}$, it is observed from Figure 1(a) for the case of very low stratification $S_{t}=0.1$, that an increase in the values of $K_{1}=K_{2}$ from 0 through $0.3,0.7$ to 1.0 corresponds to a slight increase in the velocity distribution. Under normal circumstances, it is noticed that by increasing the values of $K_{1}$ and $K_{2}$ velocity and the boundary layer thickness increase. Also, from Figure 1(b) for a high value of stratification $S_{t}=0.8$, it is noticed that as the values of $K_{1}=K_{2}$ increases, the velocity profile increases, but at the exact value of $\eta=2.65$ all profiles merge and asymptotically tends to 0 . From Figure 2(a) and Figure 2(b) it is observed that increase in $K_{1}=K_{2}$ leads to decrease in the temperature distribution for both cases of $S_{t}=0.1$ and $S_{t}=0.8$. It is noticed for the first case of stratification $S_{t}=0.1$ that as $K_{1}=K_{2}$ increases in magnitude, there is a notable decrease in the temperature profile for value of $K_{1}=K_{2}=0$ which signifies the Newtonian fluid, and thereafter the profiles decreases slightly as $K_{1}=K_{2}$ increases. Also, for the cases of $S_{t}=0.8$ in Figure 2(b) it is observed that the temperature profile substantially decreases as the values of $K_{1}=K_{2}$ increases. From Figure 3(a), it is seen that an increase in $K_{1}=K_{2}$ when $S_{t}=0.8$ corresponds to increase in the wall shear stress. Also, from Figure 3(b) it is noticed that as the $S_{t}$ increases the wall shear stress decreases for the case of the non-Newtonian fluid i.e. $K_{1}=K_{2}=1.0$. The effect of thermal stratification parameter $S_{t}$ on temperature distributions are presented in Figure 4(a) and Figure 4(b) for both cases of $K_{1}=K_{2}=0$ (Newtonian) and $K_{1}=K_{2}=1.0$ (non-Newtonian). It is depicted from the computations that for both cases of $K_{1}=K_{2}$ the temperature profiles decrease substantially as the magnitude of $S_{t}$ is increased from 0.1 through $0.4,0.7$ to 1.0 making each profile to satisfy the boundary condition associated with the model that is, $\left(\theta(\eta)=1-S_{t}\right)$. Apparently, when $S_{t}=0.1,0.4,0.7,0.9$ reflecting in the boundary condition $\left(\theta(\eta)=1-S_{t}\right)$ which results in the decrease in the value of temperature

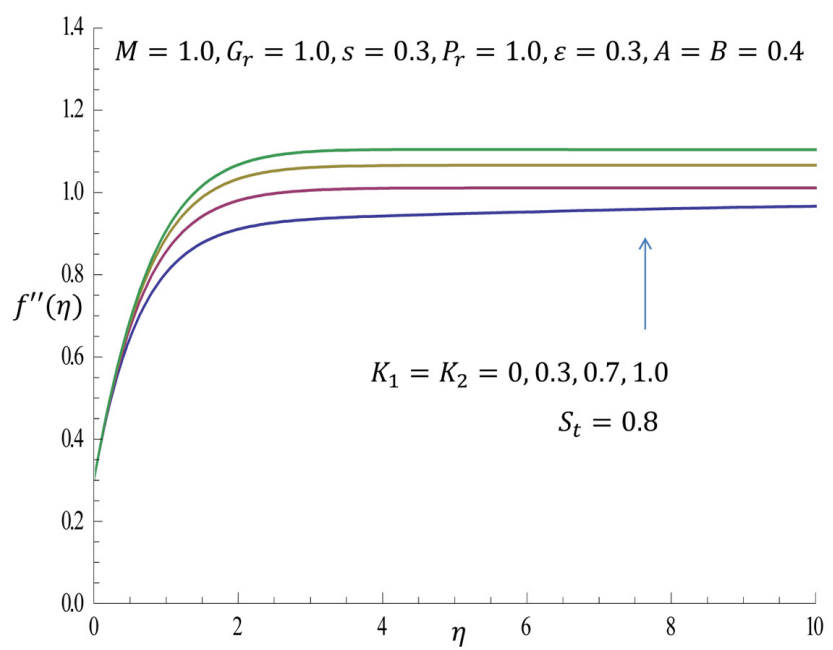

(a)

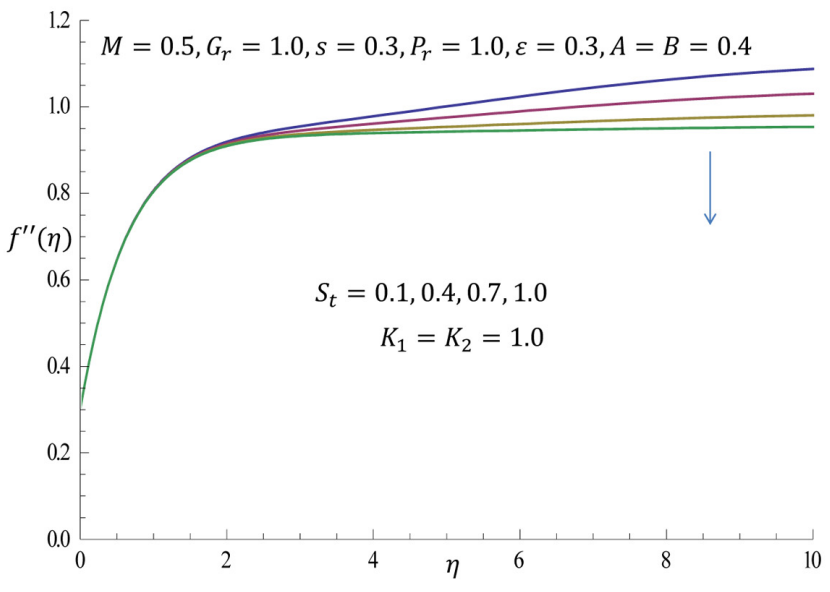

(b)

Figure 3. (a) Effect of non-Newtonian parameters $K_{1}=K_{2}$ on the wall shear stress when $S_{t}=0.8$ (b) Effect of Stratification parameter $S_{t}$ on the wall shear stress when $K_{1}=K_{2}=1.0$. 


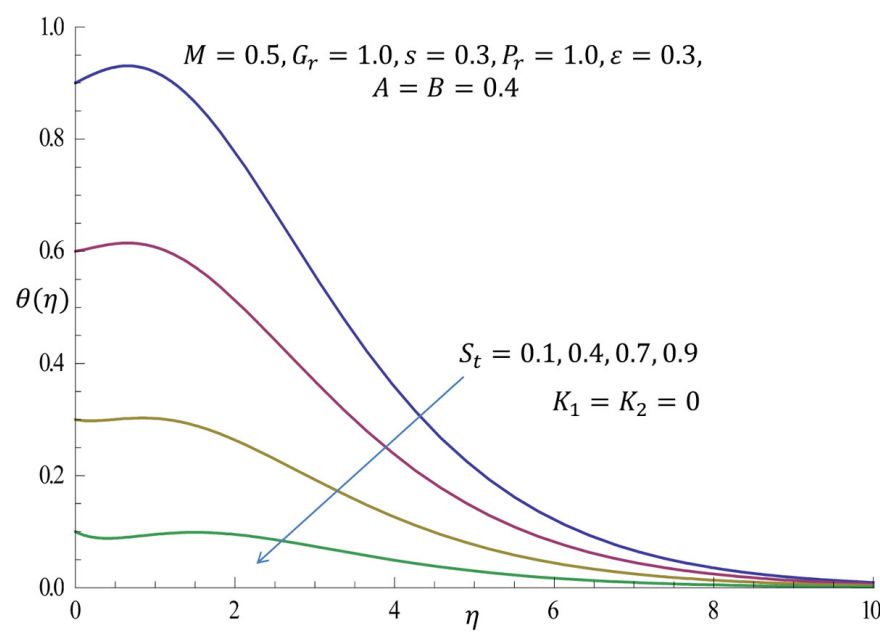

(a)

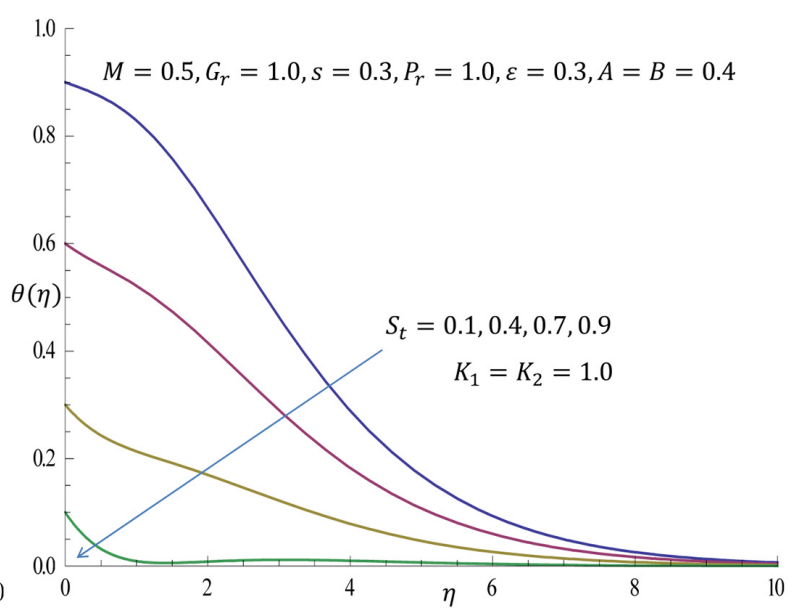

(b)

Figure 4. (a) Effect of Stratification parameter $S_{t}$ on temperature profile when $K_{1}=K_{2}=0$ (b) Effect of Stratification parameter $S_{t}$ on temperature profile when $K_{1}=K_{2}=1.0$.

$\theta(\eta)=0.9,0.6,0.3,0.1$ as revealed in the temperature profiles in Figure 4(a) and Figure 4(b). In other words, this can be traced to the fact that as $S_{t}$ increases, the surface temperature within the thermally stratified medium decreases. Physically, this decrease in the temperature distribution as the thixotropic fluid flows along a vertical surface may account for an increase in the viscosity of the fluid, and as a result of decrease in temperature the intermolecular forces binding the microstructure of thixotropic fluid becomes stronger and makes it possible for the fluid to be more viscous. Figure 5(a), Figure 5(b) and Figure 6(a), Figure 6(b) illustrate the influence of Magnetic parameter $M$ on velocity and temperature distributions respectively for different cases of non-Newtonian parameters $K_{1}=K_{2}=0.1,0.8$. It is revealed that, as $M$ increases at a fixed value of $K_{1}=K_{2}=0.1,0.8$, the velocity profiles decrease and the temperature profiles increase. Physically, this observation is due to the fact that, application of transverse magnetic field normal to the direction of the flow brings about a resistive force which has tendency to reduce the velocity of the fluid and hence makes the temperature of the fluid to rise. In order to further unravel the behaviour of the fluid, the effect of temperature-dependent thermal conductivity parameter $\varepsilon$ on temperature profiles are investigated in Figure $7(\mathrm{a})$ and Figure $7(\mathrm{~b})$ for the different cases of $K_{1}=K_{2}$ when $S_{t}=0.6$. It is observed from Figure 7(a) that increase in the values of $\varepsilon$ corresponds to increase in the temperature distribution for the case of $K_{1}=K_{2}=0$ (Newtonian) and all profiles quickly merge at $\eta=5.62$ and descend towards the free stream. Also, from Figure $7(\mathrm{~b})$ it is observed that as $\varepsilon=\delta\left(T_{w}-T_{0}\right)$ increases from 1 through $2.0,3.0$ to 4.0 for the case of $K_{1}=K_{2}=1.0$ there is an obvious increase in temperature profile within the domain $0 \leq \eta \leq 2.8$ and within the domain $2.9 \leq \eta \leq 3.7$ all profiles merge together, thereafter at specific value of $\eta=3.8$ the temperature decreases away from the surface and all profiles decay smoothly 


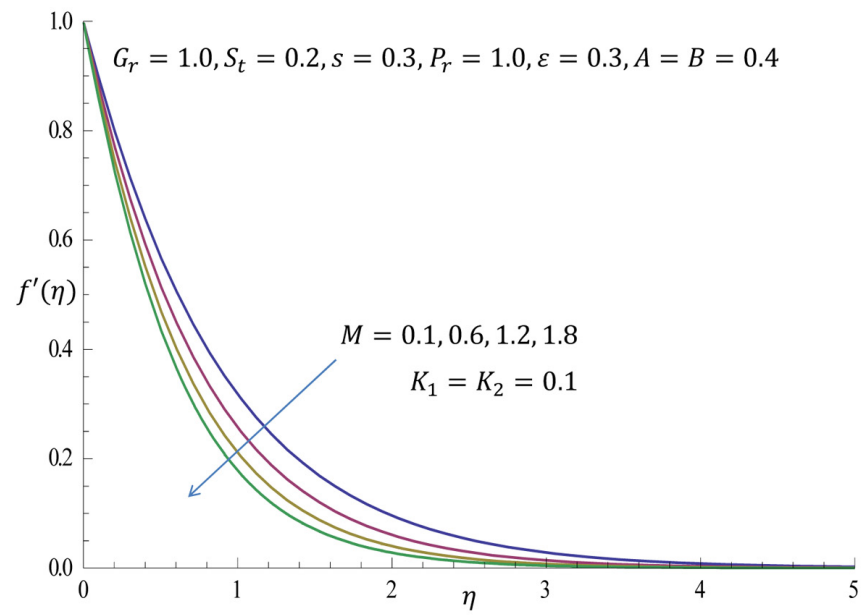

(a)

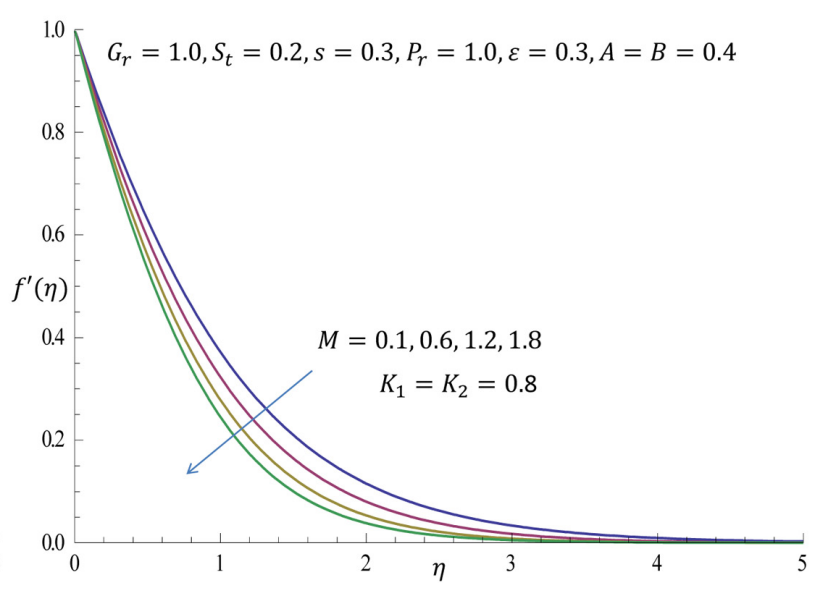

(b)

Figure 5. (a) Effect of Magnetic parameter $M$ on velocity profile when $K_{1}=K_{2}=0.1$ (b) Effect of Magnetic parameter $M$ on velocity profile when $K_{1}=K_{2}=0.8$.

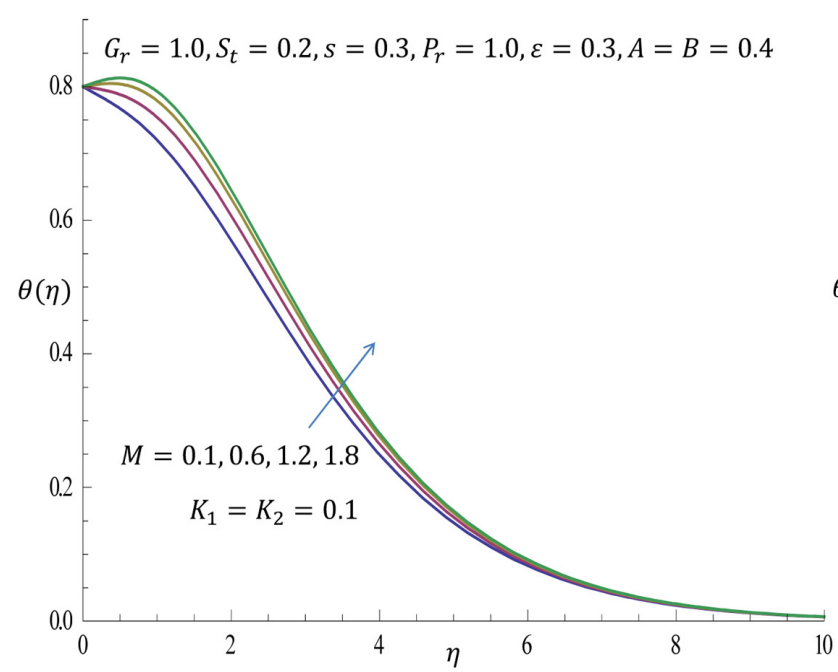

(a)

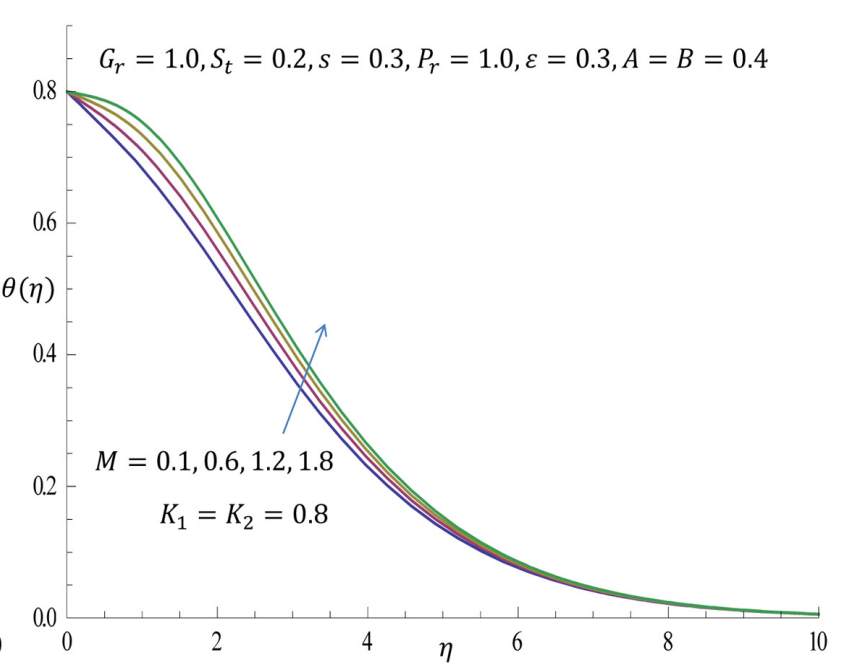

(b)

Figure 6. (a) Effect of Magnetic parameter $M$ on temperature profile when $K_{1}=K_{2}=0.1$ (b) Effect of Magnetic parameter $M$ on temperature profile when $K_{1}=K_{2}=0.8$.

to zero as $\eta \rightarrow \infty$. Figure 8 (a) and Figure $8(\mathrm{~b})$ illustrates the influence of Grashof number $G_{r}$ on the velocity and temperature profiles when $K_{1}=K_{2}=0.2$ and $S_{t}=0.4$. From Figure $8(\mathrm{a})$ as $G_{r}$ increases from 0.1 the velocity profile increases but as $G_{r}$ increases through 0.5,1.0,1.5 all profiles conflate within the domain $2.7 \leq \eta \leq 2.8$ and the velocity increases towards the free stream. Likewise, from Figure $8(\mathrm{~b})$ it is observed that increase in $G_{r}$ leads to considerable decrease in the temperature profile.

\section{Convergence of the Homotopy Solutions}

It is obvious that the series (27) and (28) consist of the non-zero auxiliary parameters $\hbar_{f}$ and $\hbar_{\theta}$ which can adjust and control the convergence. The 


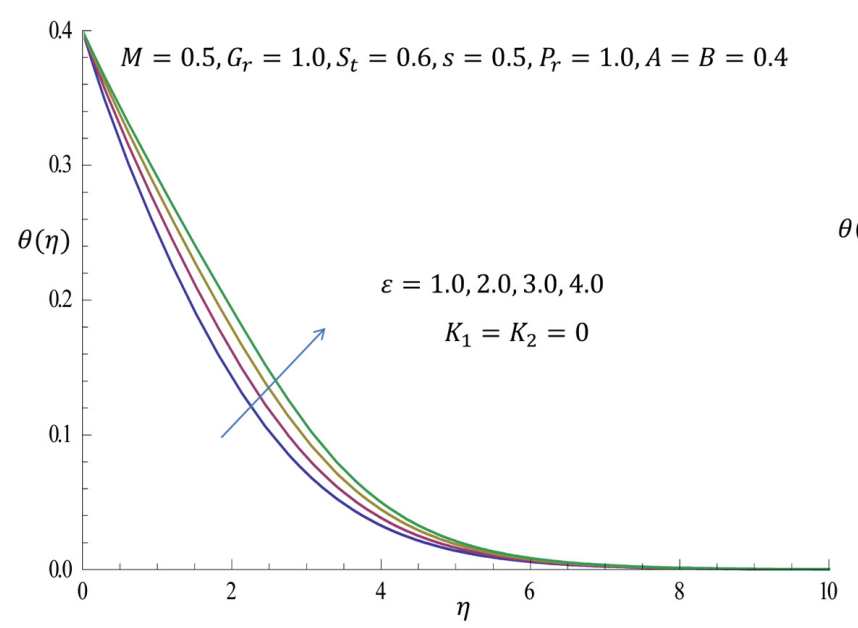

(a)

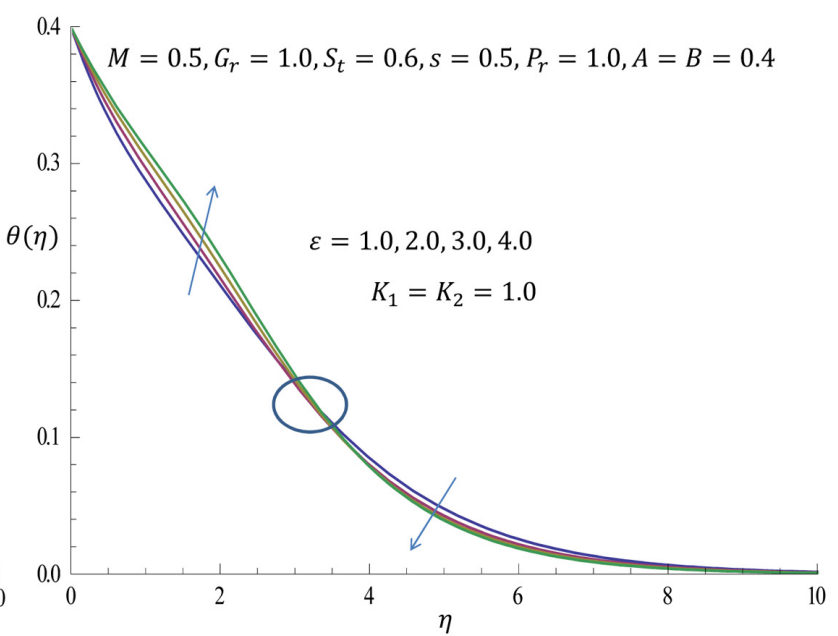

(b)

Figure 7. (a) Effect of Temperature-dependent thermal conductivity parameter $\varepsilon$ on temperature profile when $K=0$ (b) Effect of Temperature-dependent thermal conductivity parameter $\varepsilon$ on temperature profile when $K=1.0$.

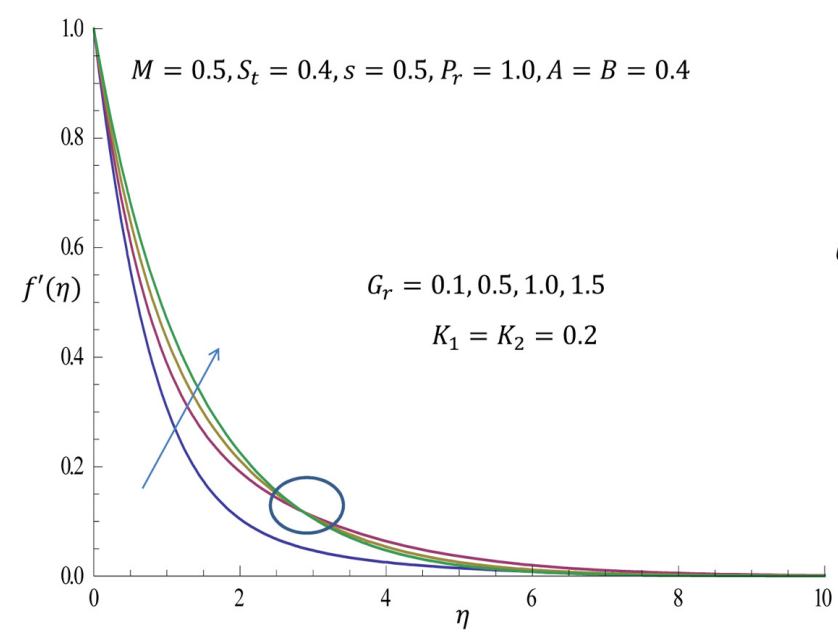

(a)

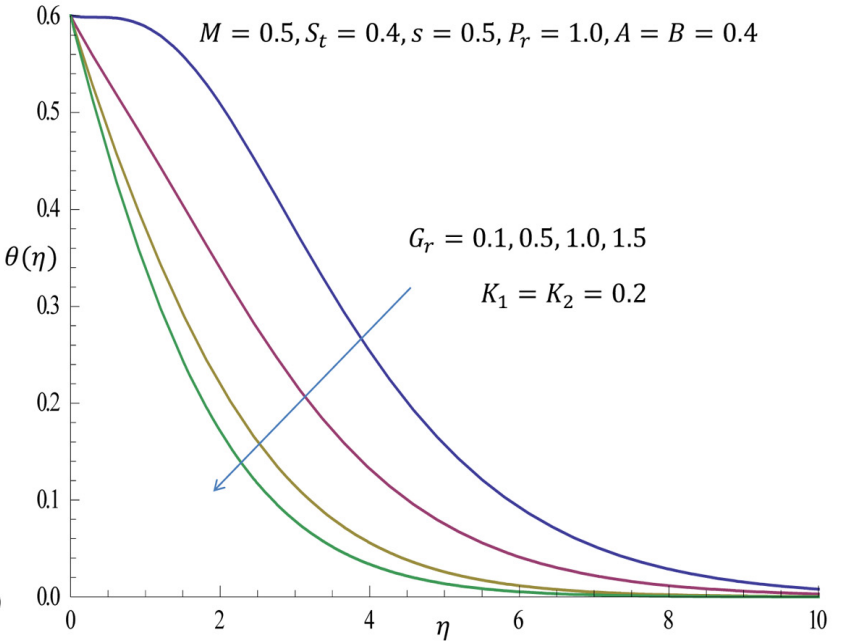

(b)

Figure 8. (a) Effect of Grashof number $G_{r}$ on velocity profile when $K_{1}=K_{2}=0.2$ (b) Effect of Grashof number $G_{r}$ on temperature profile when $K_{1}=K_{2}=0.2$.

interval on $\hbar$-axis for which the $\hbar$-curve becomes parallel to the $\hbar$-axis is recognized as the set of admissible values of $\hbar_{f}$ and $\hbar_{\theta}$ for which the solution series converges. These figures show that the ranges for the acceptable values of $\hbar_{f}$ and $\hbar_{\theta}$ are $-0.7 \leq \hbar_{f} \leq-0.4$ and $-1.3 \leq \hbar_{\theta} \leq-0.8$. Figure 9(a) and Figure 9(b) depict the $\hbar$-curves for this problem, we obtained the approximate optimal values of $\hbar_{f}$ and $\hbar_{\theta}$ at 10th-order of approximation as -0.797715 and -0.989828 .

\section{Results and Discussion}

Computation has been carried out for various values of emerging parameters like; stratification parameter, magnetic parameter, temperature dependent 


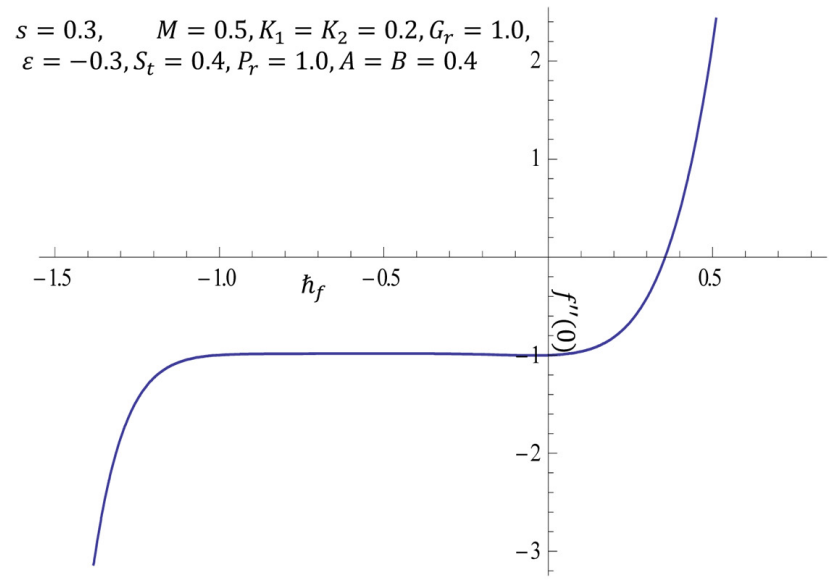

(a)

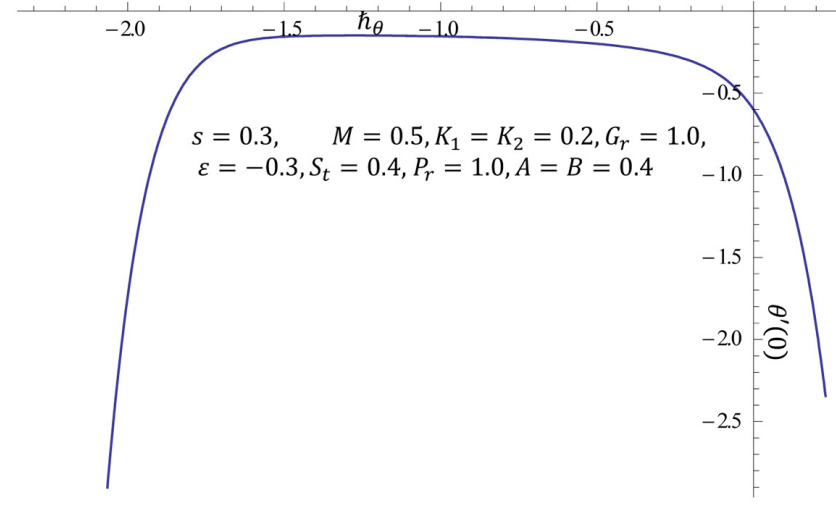

(b)

Figure 9. (a) $\hbar$-curve of $f^{\prime \prime}(0)$ obtained at 10th-order of approximation (b) $\hbar$-curve of $\theta^{\prime}(0)$ obtained at 10 th-order of approximation.

Table 1. Comparison of the results for the skin-friction coefficient $-\operatorname{Re}_{x}^{1 / 2} C_{f}$ at $M=0.6, K_{1}=0.1, \quad K_{2}=0.2$, $G_{r}=\varepsilon=S_{t}=P_{r}=A=B=0$ for various values of $s$.

\begin{tabular}{ccc}
\hline$s$ & Hayat et al. [6] & Present result \\
\hline 0 & 1.01988 & 1.03854 \\
0.7 & 1.20293 & 1.23432 \\
1.0 & 1.27055 & 1.30795 \\
\hline
\end{tabular}

Table 2. Numerical values of Skin-friction coefficient for various values $s, K_{1}$ and $K_{2}$ when $M=0.5$ and $G_{r}=0$.

\begin{tabular}{cllll}
\hline$s$ & $M$ & $K_{1}$ & $K_{2}$ & $-R e_{x}^{1 / 2} C_{f}$ \\
\hline 0.3 & 0.5 & 0.1 & 0.1 & 1.14839 \\
0.3 & 0.5 & 0.1 & 0.5 & 0.99376 \\
0.3 & 0.5 & 0.1 & 0.7 & 0.94908 \\
0.3 & 0.5 & 0.3 & 0.7 & 0.93290 \\
0.3 & 0.5 & 0.5 & 0.7 & 0.91812 \\
0.3 & 0.5 & 0.8 & 0.7 & 0.89821 \\
0.5 & 0.5 & 0.8 & 0.7 & 0.93026 \\
0.7 & 0.5 & 0.8 & 0.7 & 0.95816 \\
0.9 & 0.5 & 0.8 & 0.7 & 0.98229
\end{tabular}

thermal conductivity parameter, non-Newtonian parameters and heat source parameters. Table 1 is being validated by direct comparison with the numerical results reported by Hayat et al. [6] when $G_{r}=\varepsilon=S_{t}=A=B=0$. The numerical computations of skin-friction coefficient $-R e_{x}^{1 / 2} C_{f}$ for the values of $s, M$ and $K_{1}$ and $K_{2}$ are presented in Table 2, it is shown that the values of skin-friction coefficient $-R e_{x}^{1 / 2} C_{f}$ increase by increasing $s$ and decrease by increasing $K_{1}$ and $K_{2}$. 


\section{Conclusions}

Free convection MHD Thixotropic fluid flow past a vertical surface with temperature-dependent thermal conductivity, subjected to thermal stratification is investigated. Series solutions for the velocity and temperature fields were gotten and discussed. Effects of various parameters governing the flow fields were investigated. From this study, we conclude thus;

1) Increase in the values of $K_{1}=K_{2}$ corresponds to increase in the velocity profile for both cases of $S_{t}=0.1$ and $S_{t}=0.8$ with opposite effect of the temperature profiles.

2) The wall shear stress increases as the values of $K_{1}=K_{2}$ increase when $S_{t}=0.8$.

3) Temperature profile is a decreasing function of $S_{t}$ for both cases of $K_{1}=K_{2}=0$ and $K_{1}=K_{2}=1.0$.

4) The wall shear stress decreases with increase in values of $S_{t}$ when $K_{1}=K_{2}=1.0$.

\section{Conflicts of Interest}

The authors declare no conflicts of interest regarding the publication of this paper.

\section{References}

[1] Hendrickson, T. (2003) Massage for Orthopedic Conditions. Lippincott Williams \& Wilkins, Philadelphia, PA.

[2] Davies, M.C.R., Pender, M.J., Orense, R.P., Wotherspoon, L., Cubrinovsk, M. and Bowman, E.T. (2011) Geotechnical Implications of the M 7.1 and M 6.3 Canterbury Earthquakes of 4 September 2010 and 22 February 2011. In: Wardani, S.P.R., Chu, J., Robert Lo, S.C., Iai, S. and Phoon, K.K., Eds., Geotechnical Engineering for Disaster Mitigation and Rehabilitation and Highway Engineering 2011, World Scientific Publishing Co. Pte. Ltd., Singapore. https://doi.org/10.1142/9789814365161 0007

[3] Sadeqi, S., Khabazi, N. and Sadeghy, K. (2011) Blasius Flow of Thixotropic Fluids: A Numerical Study. Communications in Nonlinear Science and Numerical Simulation, 16, 711-721. https://doi.org/10.1016/j.cnsns.2010.05.009

[4] Harris, J. (1976) A Continuum Theory of Time-Dependent Inelastic Flow. Rheologica Acta, 6, 6-12. https://doi.org/10.1007/BF01968376

[5] Harris, J. (1977) Rheology and Non-Newtonian Flow. Longman, London.

[6] Hayat, T., Shehzad, S.A. and Asghar, S. (2013) MHD Flow of Thixotropic Fluid with Variable Thermal Conductivity and Thermal Radiation. Walailak Journal of Science and Technology, 10, 29-42.

[7] Animasaun, I.L. (2016) Double Diffusive Unsteady Convective Micropolar Flow Past a Vertical Porous Moving through a Binary Mixture Using Modified Boussinesq Approximation. Ain Shams Engineering Journal, 7, 755-765. https://doi.org/10.1016/j.asej.2015.06.010

[8] Animasaun, I.L. (2016) 47nm Alumina-Water Nanofluid Flow within Boundary Layer Formed on Upper Horizontal Surface of Paraboloid of Revolution in the 
Presence of Quartic Autocatalysis Chemical Reaction. Alexandria Engineering Journal, 55, 2375-2389. https://doi.org/10.1016/j.aej.2016.04.030

[9] Koriko, O.K., Oreyeni, T., Omowaye, A.J. and Animasaun, I.L. (2016) Homotopy Analysis of MHD Free Convective Micropolar Fluid Flow along a Vertical Surface Embedded in Non-Darcian Thermally-Stratified Medium. Open Journal of Fluid Dynamics, 6, 198-221. https://doi.org/10.4236/ojfd.2016.63016

[10] Omowaye, A.J. and Animasaun, I.L. (2016) Upper-Convected Maxwell Fluid Flow with Variable Thermo-Physical Properties over a Melting Surface Situated in Hot Environment Subject to Thermal Stratification. Journal of Applied Fluid Mechanics, 9, 1777-1790.

[11] Hayat, T., Hussain, T., Shehzad, S.A. and Alsaedi, A. (2014) Thermal and Concentration Stratifications Effects in Radiative Flow of Jeffrey Fluid over a Stretching Sheet. PLoS ONE, 9, e107858. https://doi.org/10.1371/journal.pone.0107858

[12] Abo-Eldahab, E.M. and El-Aziz, M.A. (2004) Blowing/Suction on Hydromagnetic Heattransfer by Mixed Convection from an Inclined Continuously Stretching Surface with Internal Heat Generation/Absorption. International Journal of Thermal Sciences, 43, 709-719. https://doi.org/10.1016/j.ijthermalsci.2004.01.005

[13] Muthtamilselvan, M., Prakash, D. and Doh, D.H. (2014) Effect of Non-Uniform Heat Generation on Unsteady MHD Non-Darcian Flow over a Vertical Stretching Surface with Variable Properties. Journal of Applied Fluid Mechanics, 7, 425-434.

[14] Crepeau, J.C. and Clarksean, R. (1997) Similarity Solutions of Natural Convection with Internal Heat Generation, Transactions of ASME? Journal of Heat Transfer, 119, 184-185. https://doi.org/10.1115/1.2824086

[15] Sharma, P.R. and Singh, G. (2009) Effects of Variable Thermal Conductivity and Heat Source/Sink on MHD Flow Near a Stagnation Point on a Linearly Stretching Sheet. Journal of Applied Fluid Mechanics, 2, 12-21.

[16] Uwanta, I.J. and Omokhuale, E. (2014) Effects of Variable Thermal Conductivity on Heat and Mass Transfer with Jeffery Fluid. Internal Journal of Mathematical Archive, 5, 1345-149.

[17] Prasad, K.V., Vajravelu, K. and Datti, P.S. (2010) The Effects of Variable Fluid Properties on the Hydromagnetic on the Hydromagnetic Flow and Heat Transfer over a Non-Linearly Stretching Sheet. International Journal of Thermal Sciences, 49, 603-610. https://doi.org/10.1016/j.ijthermalsci.2009.08.005

[18] Uwanta, I.J., Ahmad, S.K. and Omokhuale, E. (2014) Effect of Jeffery Fluid on Heat and Mass Transfer Past a Vertical Porous Plate with Soret and Variable Thermal Conductivity. Journal of Mathematical and Computational Science, 4, 915-939.

[19] Charraudeau, J. (1975) Influence de gradents de properties physiques en convection force application au cas du tube. International Journal of Heat and Mass Transfer, 18, 87-95. https://doi.org/10.1016/0017-9310(75)90011-3

[20] Koriko, O.K., Animasaun, I.L., Omowaye, A.J. and Oreyeni, T. (2018) The Combined Influence of Nonlinear Thermal Radiation and Thermal Stratification on the Dynamics of Micropolar Fluid along a Vertical Surface. Multidiscipline Modeling in Materials and Structures, 15, 133-155. https://doi.org/10.1108/MMMS-12-2017-0155

[21] Oyem, O.A. and Oreyeni, T. (2017) Homotopy Analysis of Magnetic Field Effect on Free Convection Flow Past a Semi-Infinite Flat Plate. ANNALS of Faculty Engineering Hunedoara-International Journal of Engineering, 4, 103-110.

[22] Hayat, T., Iqbal, Z., Mustafa, M. and Hendi, A.A. (2013) Melting Heat Transfer in the Stagnation-Point Flow of Third Grade Fluid Past a Stretching Sheet with Visc- 
ous Dissipation. Thermal Science, 17, 865-875.

https://doi.org/10.2298/TSCI110405119H

[23] Liao, S.J. (2003) Beyond Perturbation: Introduction to Homotopy Analysis Method. Chapman and Hall/CRC, New York. https://doi.org/10.1201/9780203491164

[24] Liao, S.J. (2010) An Optimal Homotopy Analysis Approach for Strongly Nonlinear Differential Equations. Communications in Nonlinear Science and Numerical Simulation, 15, 2003-2016. https://doi.org/10.1201/9780203491164 